\title{
Erratum
}

\section{Erratum to: A Note on a Symplectic Structure on the Space of $G$-Monopoles}

\author{
Michael Finkelberg $^{1}$, Alexander Kuznetsov ${ }^{1}$, Nikita Markarian ${ }^{1}$, Ivan Mirković ${ }^{2}$ \\ 1 Independent Moscow University, 11 Bolshoj Vlasjevskij Pereulok, Moscow 121002, Russia. \\ E-mail: fnklberg@gmail.com; akuznet@mi.ras.ru; nikita.markarian@gmail.com \\ 2 Dept. of Mathematics and Statistics, University of Massachusetts at Amherst, Amherst, \\ MA 01003-4515,USA.E-mail: mirkovic@math.umass.edu
}

Received: 10 March 2014 / Accepted: 18 August 2014

Published online: 21 September 2014 - C Springer-Verlag Berlin Heidelberg 2014

Commun. Math. Phys. 201, 411-421 (1999)

0.1. The calculation of the symplectic form given in the paper is correct in the simply laced case. In the non simply laced case certain corrections (due to L. Rybnikov) are in order.

To begin with, there is a certain ambiguity in the notations related to the invariant bilinear form in Sect. 3.2. To avoid a misunderstanding, let us recall that $i, j$, etc. stand for the simple coroots, alternatively denoted $\alpha_{i}, \alpha_{j}$, etc., while $i^{\prime}, j^{\prime}$, etc. stand for the corresponding simple roots, alternatively denoted $\check{\alpha}_{i}, \check{\alpha}_{j}$, etc. If we denote by $($,$) the$ unique $W$-invariant bilinear form on the weight lattice such that $\left(\check{\alpha}_{i}, \check{\alpha}_{i}\right)=2$ for a short root $\check{\alpha}_{i}$, then what is denoted by $i \cdot j$ in the paper should be replaced with $\left(\check{\alpha}_{i}, \check{\alpha}_{j}\right)$. We also set $\check{d}_{i}:=\left(\check{\alpha}_{i}, \check{\alpha}_{i}\right) / 2$.

0.2. Lemma 3 should be replaced with:

The operator $\sum \xi^{k} \otimes \xi_{k}-\left(\omega_{i}, \omega_{j}\right)$ acts as a scalar multiplication on every irreducible summand $V_{\lambda} \subset V_{\omega_{i}} \otimes V_{\omega_{j}}$.

On $V_{\omega_{i}+\omega_{j}}$ it acts as a multiplication by 0 .

If $\omega_{i}=\omega_{j}$, then on $V_{2 \omega_{i}-\check{\alpha}_{i}} \subset V_{\omega_{i}} \otimes V_{\omega_{i}}$ it acts as a multiplication by $-\left(\check{\alpha}_{i}, \check{\alpha}_{i}\right)=$ $-2 \check{d}_{i}$

If $i \neq j,\left(\check{\alpha}_{i}, \check{\alpha}_{j}\right) \neq 0$ then on $V_{\omega_{i}+\omega_{j}-\check{\alpha}_{i}-\check{\alpha}_{j}} \subset V_{\omega_{i}} \otimes V_{\omega_{j}}$ it acts as a multiplication by $\left(\left(\check{\alpha}_{i}, \check{\alpha}_{j}\right)-\frac{\left(\check{\alpha}_{i}, \check{\alpha}_{i}\right)}{2}-\frac{\left(\check{\alpha}_{j}, \check{\alpha}_{j}\right)}{2}\right)$.

Proof. Straightforward.

0.3. Lemma 4 should be replaced with:

The embedding $V_{\omega_{i}+\omega_{j}} \subset V_{\omega_{i}} \otimes V_{\omega_{j}}$ induces an isomorphism in the weights $\omega_{i}+$ $\omega_{j}, \quad \omega_{i}+\omega_{j}-\check{\alpha}_{i}, \quad \omega_{i}+\omega_{j}-\check{\alpha}_{j}, \quad \omega_{i}+\omega_{j}-\check{\alpha}_{i}-\check{\alpha}_{j}$ with the following two exceptions: 
(1) $\left(V_{\omega_{i}} \otimes V_{\omega_{i}}\right)^{2 \omega_{i}-\check{\alpha}_{i}}=V_{2 \omega_{i}-\check{\alpha}_{i}}^{2 \omega_{i}} \oplus V_{2 \omega_{i}-\check{\alpha}_{i}}^{2 \omega_{i}-\check{\alpha}_{i}}$; the G-projection to the second summand is given by the formula

$$
a\left(v_{\omega_{i}} \otimes F_{i} v_{\omega_{i}}\right)+b\left(F_{i} v_{\omega_{i}} \otimes v_{\omega_{i}}\right) \mapsto \frac{a-b}{2}\left(v_{\omega_{i}} \otimes F_{i} v_{\omega_{i}}-F_{i} v_{\omega_{i}} \otimes v_{\omega_{i}}\right)
$$

(2) $\left(V_{\omega_{i}} \otimes V_{\omega_{j}}\right)^{\omega_{i}+\omega_{j}-\check{\alpha}_{i}-\check{\alpha}_{j}}=V_{\omega_{i}+\omega_{j}}^{\omega_{i}+\omega_{j}-\check{\alpha}_{i}-\check{\alpha}_{j}} \oplus V_{\omega_{i}+\omega_{j}-\check{\alpha}_{i}-\check{\alpha}_{j}}^{\omega_{i}+\omega_{j}-\check{\check{\alpha}}_{j}-\check{\check{\alpha}}_{j}}$ if $i$ and $\left(\check{\alpha}_{i}, \check{\alpha}_{j}\right) \neq$ 0 ; the $G$-projection to the second summand is given by the formula

$$
\begin{aligned}
& a\left(v_{\omega_{i}} \otimes F_{i} F_{j} v_{\omega_{j}}\right)+b\left(F_{i} v_{\omega_{i}} \otimes F_{j} v_{\omega_{j}}\right)+c\left(F_{j} F_{i} v_{\omega_{i}} \otimes v_{\omega_{j}}\right) \\
& \mapsto \frac{b-a-c}{1-\left\langle\alpha_{i}, \check{\alpha}_{j}\right\rangle^{-1}-\left\langle\alpha_{j}, \check{\alpha}_{i}\right\rangle^{-1}}\left(\left\langle\alpha_{i}, \check{\alpha}_{j}\right\rangle^{-1} v_{\omega_{i}} \otimes F_{i} F_{j} v_{\omega_{j}}\right. \\
& \left.\quad+F_{i} v_{\omega_{i}} \otimes F_{j} v_{\omega_{j}}+\left\langle\alpha_{j}, \check{\alpha}_{i}\right\rangle^{-1} F_{j} F_{i} v_{\omega_{i}} \otimes v_{\omega_{j}}\right) .
\end{aligned}
$$

Proof. Straightforward.

0.4. Equations (7) and (8) should be replaced with

$$
\begin{aligned}
& \widetilde{D}_{\omega_{i}, \omega_{i}}^{2 \omega_{i} \check{\alpha}_{i}}=\check{d}_{i} \frac{\phi_{\omega_{i}}^{\omega_{i}}(z) \phi_{\omega_{i}}^{\omega_{i}-\check{\alpha}_{i}}(w)-\phi_{\omega_{i}}^{\omega_{i}-\check{\alpha}_{i}}(z) \phi_{\omega_{i}}^{\omega_{i}}(w)}{z-w}\left(F_{i} v_{\omega_{i}} \otimes v_{\omega_{i}}-v_{\omega_{i}} \otimes F_{i} v_{\omega_{i}}\right) \\
& \widetilde{D}_{\omega_{i}, \omega_{j}}^{\omega_{i}+\omega_{j}-\check{\alpha}_{i}-\check{\alpha}_{j}}=\frac{\phi_{\omega_{i}}^{\omega_{i}-\check{\alpha}_{i}}(z) \phi_{\omega_{j}}^{\omega_{j}-\check{\alpha}_{j}}(w)-\phi_{\omega_{i}}^{\omega_{i}}(z) \phi_{\omega_{j}}^{\omega_{j}-\check{\alpha}_{i}-\check{\alpha}_{j}}(w)-\phi_{\omega_{i}}^{\omega_{i}-\check{\alpha}_{i}-\check{\alpha}_{j}}(z) \phi_{\omega_{j}}^{\omega_{j}}(w)}{z-w} \\
& \quad \times\left(\check{\alpha}_{i}, \check{\alpha}_{j}\right)\left(\left\langle\alpha_{i}, \check{\alpha}_{j}\right\rangle^{-1} v_{\omega_{i}} \otimes F_{i} F_{j} v_{\omega_{j}}+F_{i} v_{\omega_{i}} \otimes F_{j} v_{\omega_{j}}+\left\langle\alpha_{j}, \check{\alpha}_{i}\right\rangle^{-1} F_{j} F_{i} v_{\omega_{i}} \otimes v_{\omega_{j}}\right)
\end{aligned}
$$

0.5. Proposition 2 should be replaced with:

$$
\begin{aligned}
& \left\{x_{\omega_{i}}^{k}, x_{\omega_{j}}^{l}\right\}=0 ; \\
& \left\{x_{\omega_{i}}^{k}, y_{\omega_{j}}^{l}\right\}=\check{d}_{i} \delta_{k l} \delta_{i j} y_{\omega_{j}}^{l} ; \\
& \left\{y_{\omega_{i}}^{k}, x_{\omega_{j}}^{l}\right\}=-\check{d}_{i} \delta_{k l} \delta_{i j} y_{\omega_{i}}^{k} ; \\
& \left\{y_{\omega_{i}}^{k}, y_{\omega_{j}}^{l}\right\}=\left(\check{\alpha}_{i}, \check{\alpha}_{j}\right) \frac{y_{\omega_{i}}^{k} y_{\omega_{j}}^{l}}{x_{\omega_{i}}^{k}-x_{\omega_{j}}^{l}}, \quad \text { if } i \neq j ; \\
& \left\{y_{\omega_{i}}^{k}, y_{\omega_{i}}^{l}\right\}=0 .
\end{aligned}
$$

0.6. Remark 2 should be replaced with:

The matrix of the bivector field $P^{\mathbf{X}}$ in the coordinates $\left(x_{\omega_{i}}^{k}, y_{\omega_{i}}^{k}\right)$ looks as follows

$$
\left(\begin{array}{c|c}
0 & \operatorname{diag}\left(\check{d}_{i} y_{\omega_{i}}^{k}\right) \\
\hline-\operatorname{diag}\left(\check{d}_{i} y_{\omega_{i}}^{k}\right) & *
\end{array}\right)
$$

Since on the open set $U$ this matrix is evidently nondegenerate it follows that the functions $\left(x_{\omega_{i}}^{k}, y_{\omega_{i}}^{k}\right)$ indeed form an étale coordinate system. 
0.7. Remark 3 should be replaced with:

One can easily write down the corresponding symplectic form in the coordinates:

$$
\sum_{i, k} \check{d}_{i} \frac{d y_{\omega_{i}}^{k} \wedge d x_{\omega_{i}}^{k}}{y_{\omega_{i}}^{k}}+\frac{1}{2} \sum_{i \neq j}\left(\check{\alpha}_{i}, \check{\alpha}_{j}\right) \frac{d x_{\omega_{i}}^{k} \wedge d x_{\omega_{j}}^{l}}{x_{\omega_{i}}^{k}-x_{\omega_{j}}^{l}}
$$

0.8. The last line of Subsect. 1.2 should be replaced with:

$\left\{x_{i}^{k}, x_{j}^{l}\right\}=0=\left\{y_{i}^{k}, y_{i}^{l}\right\} ;\left\{x_{i}^{k}, y_{j}^{l}\right\}=\check{d}_{i} \delta_{i j} \delta_{k l} y_{j}^{l} ;\left\{y_{i}^{k}, y_{j}^{l}\right\}=\left(\check{\alpha}_{i}, \check{\alpha}_{j}\right) \frac{y_{i}^{k} y_{j}^{l}}{x_{i}^{k}-x_{j}^{l}}$ for $i \neq j$. 